\title{
Deutsche Bibliotheksstatistik (DBS) erfasst ab 2021 Daten zu Schulbibliotheken in Deutschland
}

https://doi.org/10.1515/bd-2021-0029

Ab 2021 wird die Deutsche Bibliotheksstatistik (DBS) Daten zu den Schulbibliotheken in Deutschland erfassen. Damit wird es nach intensiven Bestrebungen der Fachkommission Bibliothek und Schule des Deutschen Bibliotheksverbandes e.V. (dbv) vom Hochschulbibliothekszentrum (hbz) des Landes Nordrhein-Westfalen erstmals ermöglicht, dass wichtige Daten zu den Aufgaben, Strukturen und zur Nutzung von Schulbibliotheken erhoben werden können, um so ihr Bildungspotential sichtbar zu machen.

Das Schulbibliothekswesen in Deutschland ist sehr heterogen. So gibt es die Schulbibliothek als Zweigstelle einer Öffentlichen Bibliothek, als Schulbibliotheks-Verbund mit unterschiedlichen organisierenden Institutionen, als kombinierte Öffentliche Bibliothek und Schulbibliothek, oder als selbständige Schulbibliothek, bei der die Schule die Bibliothek eigenständig betreibt.

Dazu Prof. Dr. Andreas Degkwitz, Bundesvorsitzender des dbv: „Dank unserer Kommission Bibliothek und Schule und der gelungenen Kooperation mit dem hbz sind wir einen entscheidenden Schritt vorangekommen, das große Potential der Schulbibliotheken nun auch statistisch abzubilden. Denn Schulbibliotheken leisten einen wichtigen Beitrag zur Förderung der Lese-, Medien- und Informationskompetenz von Schüler`innen. Die erhobenen Daten werden zudem eine wichtige Grundlage für die strategischen Planungen von Politik, Schulträgern und Verbänden bilden und sichtbar machen, welch große Leistung die Schulbibliotheken als Bildungspartner erbringen.“

Dr. Silke Schomburg, Leiterin des hbz, führt aus: „Das hbz stellt für die Erhebung der Schulbibliotheks-Daten zum einen eine leistungsfähige technische Plattform zu Verfügung, zum anderen werden die Mitarbeiterinnen der DBS-Redaktion als Ansprechpersonen die Anmeldung der Schulbibliotheken in diesem Jahr begleiten. Mit der Eingabe möglichst vieler Schulbibliotheksdaten für das Berichtsjahr 2021 wird das Datenset der nationalen Bibliothekstatistik DBS künftig komplettiert und die internationalen Statistiken ergänzt werden.“

Im Frühjahr 2021 können sich die Schulbibliotheken bei der DBS anmelden. Im Laufe des Jahres 2021 können sie dann die gewünschten Daten in ihrer Institution erheben und ab dem 1. Januar 2022 für das Jahr 2021 eingeben. Die ersten Auswertungen werden im Frühjahr 2022 erwartet.

Weiterführende Informationen in Kürze auf www.schulmediothek.de. 


\section{Zum Hintergrund}

Bislang fehlte eine deutschlandweite Erhebung zur Quantität und zur Qualität von Schulbibliotheken. Bereits 2015 hat sich der Deutsche Bibliotheksverband in seiner Strategie 2016-2020 dafür ausgesprochen, sich für den strukturierten Aufbau des Schulbibliothekssystems in den Bundesländern einzusetzen. Auch die Internationale Vereinigung bibliothekarischer Verbände und Einrichtungen (IFLA) fordert die Mitgliedsländer auf, ihre Daten zu Schulbibliotheken in die „Weltkarte der Bibliotheken“ aufzunehmen.

An der Arbeitsgruppe der dbv-Kommission Bibliothek \& Schule zur Einrichtung der entsprechenden statistischen Abfrage waren beteiligt: Irene Säckel von der Stadtbücherei Frankfurt am Main, Frank Raumel vom Medien- und Informationszentrum Biberach, Ira Foltin, Gaby Heugen-Ecker und Therese Nap von der DBS-Redaktion des Hochschulbibliothekszentrums des Landes NRW sowie Dr. Ulla Wimmer von der Humboldt Universität zu Berlin.

\section{Der Deutsche Bibliotheksverband e.V. (dbv)}

Der Deutsche Bibliotheksverband e.V. (dbv) vertritt mit seinen mehr als 2.100 Mitgliedern bundesweit über 9.000 Bibliotheken mit 25.000 Beschäftigten und 11 Mio. Nutzer^innen. Sein zentrales Anliegen ist es, Bibliotheken zu stärken, damit sie allen Bürger ${ }^{\star}$ freien Zugang zu Informationen ermöglichen. Der Verband setzt sich ein für die Entwicklung innovativer Bibliotheksleistungen für Wissenschaft und Gesellschaft. Als politische Interessensvertretung unterstützt der dbv die Bibliotheken insbesondere auf den Feldern Informationskompetenz und Medienbildung, Leseförderung und bei der Ermöglichung kultureller und gesellschaftlicher Teilhabe für alle Bürgerinnen.

Mehr Informationen finden Sie unter www.bibliotheksverband.de.

\section{Pressekontakt}

Kristin Bäßler

Deutscher Bibliotheksverband

Leitung Kommunikation / Pressesprecherin

Tel.: + 49 (0)30 6449899 25 | E-Mail: baessler@bibliotheksverband.de 